\title{
Optimization of Regional City Economic Gravity Network Based on Optimal Tree Theory
}

\author{
Xin Wei*, Liang Ma, Yong Liu, Huizhen Zhang \\ School of Management, University of Shanghai for Science and Technology, Shanghai 200093, China
}

Corresponding Author Email: xinwei@usst.edu.cn

https://doi.org/10.18280/ijsdp.150619

Received: 8 April 2020

Accepted: 12 July 2020

\section{Keywords:}

regional city network, gravity model, network optimization, optimal tree

\begin{abstract}
Inspired by the gravity model of spatial economics, this paper sets up an economic gravity network for the cities in the same region, and optimizes the established network by the optimal tree theory of operations research, rather than the traditional social network analysis. After that, the research method was applied to build and optimize the economic gravity network of the cities in the Yangtze River Delta, based on the key indices of these cities in 2018. On this basis, policy recommendations were provided in terms of resource utilization, sustainable design, and industrial distribution, shedding new lights on sound policy-making, rational resource flow, and integral development of the region. The research findings lay a solid theoretic basis for regional planning and national development.
\end{abstract}

\section{INTRODUCTION}

The integration of global economy has strengthened the connections between cities, creating regional city networks. Taking China for instance, three well-known regional city networks have formed, namely, Beijing-Tianjin-Hebei (BTH) region, Yangtze River Delta, and Peral River Delta. These regional city networks become major growth poles of national economy, and occupy important positions in the strategic layout of the country.

Many Chinese scholars have explored deep into regional city networks. For example, Peng [1] investigated the overall features and spatial structure of the cities in and around the Guangdong-Hong Kong-Macao Greater Bay Area. Lao et al. [2] combined the gravity model and social network analysis to measure the economic connections among cities in the city clusters along the middle reaches of the Yangtze River. Zhao [3] studied the spatial network and organizational features of the cities in the Yangtze River Delta, based on innovation gravity and the degree of connection to external innovators. From the massive data on netizen behaviors, Wang and Zhao [4] established a Baidu index-sharing platform to evaluate network connections between cities in the era of mobile Internet.

From the global perspective, Camagni [5] was the first to extend city-level research to the level of regional city network. Since then, many foreign scholars have explored regional city networks from various angles. From the perspective of capital, Garcia and Chavez [6] established a framework based on knowledge interaction, and expounded on the innovation system of the Monterrey regional city network in Mexico. Abelem and Stanton [7] enumerated the network features of major cities in the Amazon Basin of Brazil, and analyzed different plans for building facility networks under a specific model. In the context of the booming economy in China from 2010 to 2016 , Derudder et al. calculated the connectivity of major Chinese cities in a global city network, evaluated the state changes of these cities in the network, and found out the internal and external causes of such changes [8,9].

Regional city network has evolved into an important frontier in the research on the relationship between cities. Most of the existing studies on regional city networks are qualitative or empirical analyses on the relationship between a central city and other cities in a regional city network. The relevant scholars either put forward suggestions through social network analysis, or explored the spatiotemporal evolution of the network structure induced by the changes in the function and scale of cities.

This paper sets up a regional city economic gravity network based on the gravity model of spatial economics, and optimize the network by the optimal tree theory of operations research. Following this theory, the maximum spanning tree was converted into the classical minimum spanning tree (MST), which is better aligned with the optimization task of the regional city economic gravity network. Then, the research method was tested based on the data on the cities in the Yangtze River Delta. The research results provide a good reference for policymakers to optimize regional city network and make comprehensive regional plans.

\section{CONSTRUCTION OF REGIONAL ECONOMIC GRAVITY NETWORK}

\subsection{Economic gravity model}

The mass and distance factors in the basic gravity model were modified to reflect the actual economic connections among cities [10-14]. Let $E M_{i}$ and $E M_{j}$ be the economic qualities of cities $i$ and $j$, respectively, and $E R$ be the economic distance between the two cities. Then, the modified economic gravity model between cities can be expressed as: 


$$
F_{i j}=k \frac{E M_{i} \times E M_{j}}{E R_{i j}{ }^{2}}
$$

where, $k=1$ is the gravitation constant; $E M$ is a series of economic data of the city. The above indices were organized into a multidimensional evaluation system. In addition, the $\mathrm{n}$ transportation modes between cities were considered, and assigned different weights according to the actual situation. Let $\alpha_{i j}^{f}$ be the weight of the $f$-th transportation mode between cities $\mathrm{i}$ and $\mathrm{j}$, and $T_{i j}^{f}$ and $C_{i j}^{f}$ be the time cost and monetary cost of the $f$-th transportation mode between cities $i$ and $j$. Then, the improved economic distance $E R_{i j}$ between the two cities can be expressed as:

$$
E R_{i j}=\left(\sum_{f=1}^{n} \alpha_{i j}^{f} T_{i j}^{f} C_{i j}^{f}\right)^{\frac{1}{2}}
$$

\subsection{Network construction}

Jefferson and Zipf were the first to apply the gravity model to examine the interaction between cities in a regional city network. Since then, many studies have shown that the economic gravity model is suitable to reveal the spatial relationship between two cities. Based on the economic gravity model between cities, the graph theory in operations research was adopted to construct a regional city network.

Let $G=(V, E, F)$ be a weighted graph, where $V=$ $\{1,2, \cdots, n\}$ is the set of $\mathrm{n}$ vertices (the $\mathrm{n}$ cities in the region), $E=\left\{e_{1}, e_{2}, \cdots, e_{m}\right\}$ is the set of edges (economic connections between cities in the region), and $F=\left(f_{i j}\right)_{n \times n}$ is the weighted matrix between vertexes $\left(f_{i j}>0, f_{i i}=+\infty, i, j \in V\right)$, indicating the economic gravity fromed by logistics, human flow, capital flow, and technology flow. If the vertices of edge $e_{k}$ are $i$ and $j$, then $e_{k}=(i, j)$, which indicates the economic connection between cities $i$ and $j$.

On the regional level, each resource element circulates freely across between all cities in the region, creating a regional city network.

\section{NETWORK OPTIMIZATION BASED ON OPTIMAL TREE THEORY}

\subsection{Network optimization model}

Minimum spanning tree (MST) $[15,16]$ is a basic concept of network optimization in operations research. It is designed to find a spanning three with minimum total cost for a given weighted graph. Hence, the MST has been frequently adopted for network optimization to solve engineering problems [1720]. On the contrary, the maximum spanning tree aims to find a spanning tree with maximum total cost, which enhances the benefit and impact of the network [21,22].

In the previous section, a regional city network was created based on the economic gravity model, in which all resource elements circulate freely across the region under economic gravity $F_{i j}$. From the perspective of circulation, the overall effect of the network should be maximized through optimal allocation of regional resources. Therefore, the economic gravity network can be optimized by solving its maximum spanning tree:

$$
\begin{gathered}
\max Z(x)=\sum_{i=1}^{n} \sum_{j=1}^{n} f_{i j} x_{i j} \\
\sum_{i=1}^{n} \sum_{j=1}^{n} x_{i j}=n-1 \\
\sum_{i \in S} \sum_{j \in S} x_{i j} \leq|S|-1, \quad \forall S \subset V, S \neq \varnothing \\
x_{i j} \in\{0,1\}
\end{gathered}
$$

where, $x_{i j}$ is 1 if the edge $(i, j)$ falls in the optimized network; $x_{i j}$ is 0 if otherwise; Constraint (4) is the requirements of the spanning tree; Constraint (5) prevents the generation of subloops in the spanning tree by controlling the number of vertices $|S|$ of graph $G$ in set $S$.

The objective function (3) ensures that the spanning tree with the maximum economic gravity can be obtained to maximize the overall effect of the network.

\subsection{Optimal solution to the network}

The MST problem can be solved effectively with many mature and efficient algorithms within polynomial time, such as the classic Prim algorithm and Kruskal algorithm [23, 24]. The maximum spanning tree of the network can be converted into an MST problem by constructing the equivalent network $G^{\prime}=\left(V, E, F_{i j}{ }^{\prime}\right)$ of the original network $G=(V, E, F)$.

According to the Prim algorithm, the following steps were designed to solve the maximum spanning tree of the regional city economic gravity network, that is, to find the optimal path that maximizes the long-term resource flow between cities in the region:

Step 1. Import the regional city economic gravity network graph $G^{\prime}$ and the improved economic gravity matrix $F^{\prime}[i, j]$.

Step 2. Initialize and output the total weight of the optimal tree tweight $\leftarrow 0$, the endpoint arrays of the corresponding spanning tree tedge 1 ,tedge $2=0$, the number of edges tcount $\leftarrow 0$, the nearest neighbor array nearest $\leftarrow 0$, and the gravity array dist $\leftarrow 0$.

Step 3. For $i \leftarrow 2$ to $n$, nearest $[i] \leftarrow 1$ and $\operatorname{dist}[i] \leftarrow$ $F^{\prime}[i, j]$.

Step 4. The minimum value in $\min \leftarrow$ dist,$u \leftarrow$ the selected minimum gravitational point.

Step 5. For tcount $\leftarrow$ tcount +1 , tweight $\leftarrow$ tweight + $\operatorname{dist}[u]$, update the endpoint arrays of the spanning tree as tedge 1 [tcount $] \leftarrow$ nearest $[u]$, tedge $2[$ tcount $] \leftarrow u$, and then earest $[u] \leftarrow 0$.

Step 6. For $k \leftarrow 2$ to $n$, update the nearest neighbor array nearest and the gravity array dist; If $F^{\prime}[k$, nearest $[k]]>$ $F^{\prime}[k, u]$, then $\operatorname{dist}[k] \leftarrow F^{\prime}[k, u]$ and nearest $[k] \leftarrow u$.

Step 7. If tcount $<n-1$, go back to Step 4; Otherwise, skip to Step 8.

Step 8. Output the total weight of the optimal tree tweight and the endpoint arrays of the corresponding edges tedge1, tedge2.

It is easy to see that the computational complexity of this algorithm is $O\left(n^{2}\right)$. Then, a program was compiled and ran successfully on Windows 10 , using the programming language Embarcadero Delphi. 


\section{CASE ANALYSIS}

\subsection{Optimization of the regional city economic gravity} network of the Yangtze River Delta

The case analysis focuses on typical cities in the Yangtze River Delta, including Shanghai, Nanjing, Zhenjiang, Nantong, Suzhou, Wuxi, Yangzhou, Zhenjiang, Taizhou, Hangzhou, Huzhou, and Jiaxing. The 2018 data on multiple indices were obtained for each city, covering population, employment, and economy.

Referring to the relevant literature, the economic quality of each city was measured by a total of 16 indices, such as employment, gross domestic product (GDP), fiscal revenue, import, export, to name but a few. The relevant data were extracted from statistical yearbooks released by these cities in 2019, following the principles of comparability, representativeness, continuity, and availability.

Next, the Statistical Package for the Social Sciences (SPSS) was adopted to extract the principal factors FAC1 and FAC2 whose eigenvalues are greater than 1 . Taking variance contribution rate as the weight, the economic quality of each city in the Yangtze River Delta was evaluated. For convenience, the economic qualities were adjusted to positive values (Table 1) through translation and magnification.

Following a comprehensive evaluation of the various transportation modes among cities in the Yangtze River Delta, the time $\operatorname{cost} T_{i j}^{f}$ and monetary $\operatorname{cost} C_{i j}^{f}$ of travelling between two cities were simulated by the intercity travel time using various transport modes and the cost of various transport modes, respectively, aiming to measure the economic distance between every two cities realistically. The relevant data were downloaded from the official website of the Chinese Ministry of Transport to compute the time and monetary costs. The weight $\alpha_{i j}^{f}$ of each transport mode was set according to the current transportation situation in the Yangtzer River Delta and the ratio of highways to railways (6: 4). The economic distance between every two cities in the Yangtze River Delta was measured by formula (2) (Table 2).

After that, the economic gravity $F_{i j}$ between cities was derived from the economic qualities and distances measured above. The economic gravity matrix of cities in the Yangtze River Delta was thereby constructed (Table 3).

Using the proposed optimization algorithm for regional city economic gravity network, the maximum spanning tree $T_{\max }$, which has the maximum total economic gravity of the regional city network in the Yangtze River Delta, was obtained as Figure 1. $T_{\max }$ is the optimization solution that maximizes the total economic gravity of cities in the Yangtze River Delta. In other words, $T_{\max }$ is the key gravitational path to maximize the long-term circulation of resource elements, including human, financial, material, and information resources, among cities in the region.

Table 1. The economic qualities of the cities in the Yangtze River Delta

\begin{tabular}{ccccc}
\hline City & FAC1 & FAC2 & $\begin{array}{c}\text { Economic } \\
\text { quality, } \\
\text { EM }\end{array}$ & $\begin{array}{c}\text { Adjusted } \\
\text { economic } \\
\text { quality, EM }\end{array}$ \\
\hline Shanghai & 1.860 & 1.903 & 1.759 & 23.594 \\
Nanjing & -1.147 & 1.894 & 0.054 & 6.540 \\
Wuxi & 0.241 & -0.473 & -0.039 & 5.608 \\
Changzhou & -0.274 & -0.420 & -0.311 & 2.892 \\
Suzhou & 2.219 & -1.049 & 0.867 & 14.668 \\
Nantong & -0.124 & -0.366 & -0.206 & 3.941 \\
Yangzhou & -0.451 & -0.458 & -0.425 & 1.745 \\
Zhenjiang & -0.670 & -0.360 & -0.513 & 0.873 \\
Taizhou & -0.437 & -0.506 & -0.435 & 1.646 \\
Hangzhou & -0.299 & 0.962 & 0.188 & 7.876 \\
Jiaxing & -0.191 & -0.590 & -0.327 & 2.726 \\
Huzhou & -0.667 & -0.537 & -0.577 & 0.232 \\
\hline
\end{tabular}

Table 2. The economic distance matrix of cities in the Yangtze River Delta

\begin{tabular}{ccccccc}
\hline City & Shanghai & Nanjing & Wuxi & Changzhou & Suzhou & Nantong \\
\hline Shanghai & 0 & 29.214 & 13.947 & 17.598 & 10.95 & 15.849 \\
Nanjing & 29.214 & 0 & 18.848 & 12.759 & 21.058 & 25.323 \\
Wuxi & 13.947 & 18.848 & 0 & 6.594 & 4.253 & 15.089 \\
Changzhou & 17.598 & 12.759 & 6.594 & 0 & 9.312 & 18.547 \\
Suzhou & 10.95 & 21.058 & 4.253 & 9.312 & 0 & 13.122 \\
Nantong & 15.849 & 25.323 & 15.089 & 18.547 & 13.122 & 0 \\
Yangzhou & 34.062 & 10.279 & 20.317 & 14.074 & 23.321 & 16.165 \\
Zhenjiang & 24.2 & 7.521 & 32.301 & 7.352 & 15.179 & 19.478 \\
Taizhou & 27.812 & 15.442 & 17.527 & 11.011 & 18.983 & 11.702 \\
Hangzhou & 16.966 & 27.71 & 20.371 & 22.092 & 16.904 & 28.237 \\
Jiaxing & 10.667 & 29.775 & 13.753 & 17.802 & 10.041 & 20.471 \\
Huzhou & 17.015 & 20.499 & 17.019 & 16.24 & 10.557 & 22.336 \\
\hline City & Yangzhou & Zhenjiang & Taizhou & Hangzhou & Jiaxing & Huzhou \\
\hline Shanghai & 34.062 & 24.2 & 27.812 & 16.966 & 10.667 & 17.015 \\
Nanjing & 10.279 & 7.521 & 15.442 & 27.71 & 29.775 & 20.499 \\
Wuxi & 20.317 & 32.301 & 17.527 & 20.371 & 13.753 & 17.019 \\
Changzhou & 14.074 & 7.352 & 11.011 & 22.092 & 17.802 & 16.24 \\
Suzhou & 23.321 & 15.179 & 18.983 & 16.904 & 10.041 & 10.557 \\
Nantong & 16.165 & 19.478 & 11.702 & 28.237 & 20.471 & 22.336 \\
Yangzhou & 0 & 6.358 & 6.967 & 35.798 & 32.806 & 27.3 \\
Zhenjiang & 6.358 & 0 & 9.096 & 27.839 & 23.538 & 22.65 \\
Taizhou & 6.967 & 9.096 & 0 & 35.6 & 27.62 & 26.879 \\
Hangzhou & 35.798 & 27.839 & 35.6 & 0 & 8.762 & 8.523 \\
Jiaxing & 32.806 & 23.538 & 27.62 & 8.762 & 0 & 10.076 \\
Huzhou & 27.3 & 22.65 & 26.879 & 8.523 & 10.076 & 0 \\
\hline
\end{tabular}




\subsection{Results analysis}

Based on the economic gravities among the cities in the Yangtze River Delta, the following results can be obtained from the optimization solution to the regional city economic gravity network:
(1) Shanghai boasted the best economic strength in the Yangtze River Delta. This dominant advantage is confirmed by the optimization solution to the network. In the obtained maximum spanning tree $T_{\max }$, Shanghai directly radiated eight $(67 \%)$ cities in the region.

Table 3. The economic gravity matrix of cities in the Yangtze River Delta

\begin{tabular}{ccccccc}
\hline City & Shanghai & Nanjing & Wuxi & Changzhou & Suzhou & Nantong \\
\hline Shanghai & 0 & 5.282 & 9.486 & 3.878 & 31.605 & 5.866 \\
Nanjing & 5.282 & 0 & 1.946 & 1.483 & 4.556 & 1.018 \\
Wuxi & 9.486 & 1.946 & 0 & 2.460 & 19.337 & 1.464 \\
Changzhou & 3.878 & 1.483 & 2.460 & 0 & 4.556 & 0.615 \\
Suzhou & 31.605 & 4.556 & 19.337 & 4.556 & 0 & 4.405 \\
Nantong & 5.866 & 1.018 & 1.464 & 0.615 & 4.405 & 0 \\
Yangzhou & 1.209 & 1.110 & 0.482 & 0.359 & 1.098 & 0.425 \\
Zhenjiang & 0.851 & 0.759 & 0.152 & 0.344 & 0.844 & 0.177 \\
Taizhou & 1.396 & 0.697 & 0.527 & 0.432 & 1.272 & 0.554 \\
Hangzhou & 10.953 & 1.859 & 2.168 & 1.031 & 6.834 & 1.099 \\
Jiaxing & 6.029 & 0.599 & 1.111 & 0.443 & 3.982 & 0.525 \\
Huzhou & 0.321 & 0.074 & 0.076 & 0.041 & 0.322 & 0.041 \\
\hline City & Yangzhou & Zhenjiang & Taizhou & Hangzhou & Jiaxing & Huzhou \\
\hline Shanghai & 1.209 & 0.851 & 1.396 & 10.953 & 6.029 & 0.321 \\
Nanjing & 1.110 & 0.759 & 0.697 & 1.859 & 0.599 & 0.074 \\
Wuxi & 0.482 & 0.152 & 0.527 & 2.168 & 1.111 & 0.076 \\
Changzhou & 0.359 & 0.344 & 0.432 & 1.031 & 0.443 & 0.041 \\
Suzhou & 1.098 & 0.844 & 1.272 & 6.834 & 3.982 & 0.322 \\
Nantong & 0.425 & 0.177 & 0.554 & 1.099 & 0.525 & 0.041 \\
Yangzhou & 0 & 0.240 & 0.412 & 0.384 & 0.145 & 0.015 \\
Zhenjiang & 0.240 & 0 & 0.158 & 0.247 & 0.101 & 0.009 \\
Taizhou & 0.412 & 0.158 & 0 & 0.364 & 0.162 & 0.014 \\
Hangzhou & 0.384 & 0.247 & 0.364 & 0 & 2.450 & 0.214 \\
Jiaxing & 0.145 & 0.101 & 0.162 & 2.450 & 0 & 0.063 \\
Huzhou & 0.015 & 0.009 & 0.014 & 0.214 & 0.063 & 0 \\
\hline & & & & & &
\end{tabular}

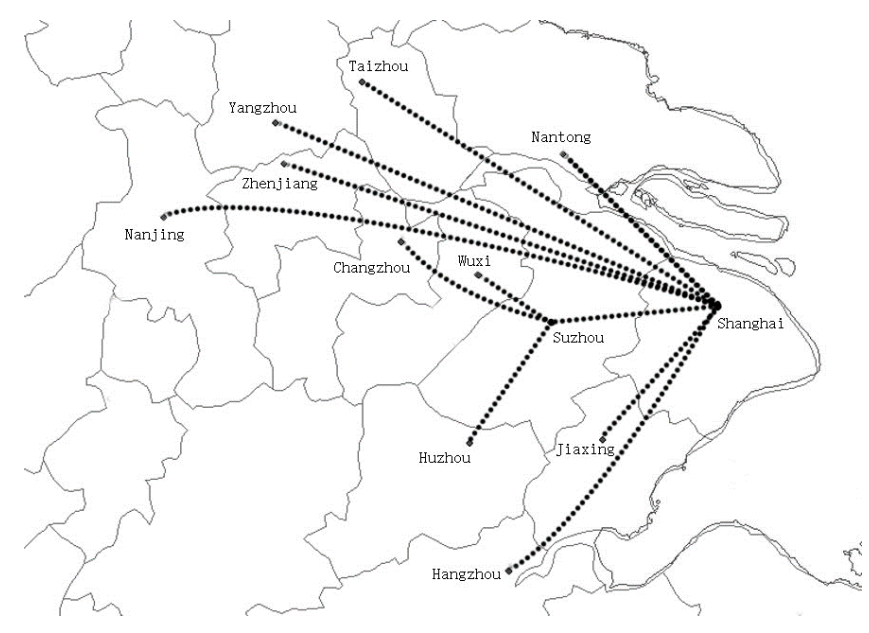

Figure 1. The optimal tree of the economic gravity network in the Yangtze River Delta

(2) Suzhou ranked second only to Shanghai in terms of economic quality, even outdoing provincial capitals like Nanjing and Hangzhou. In obtained maximum spanning tree $T_{\max }$, Suzhou existed as an economic subcenter with a small radiation range. The city directly radiated Wuxi, Changzhou, and Huzhou.

(3) As indicated by the optimization solution $T_{\max }$, Nanjing and Hangzhou, which are widely recognized by traditional research as the two wings of economic growth in the region, did not have apparent advantages over other cities. Their dominant positions were challenged by a rising star: Suzhou. Suzhou had overtaken Nanjing and Hangzhou in total economic output, and successfully developed a high-tech park economy, by virtue of its short economic distance from Shanghai. Therefore, many industrial and manufacturing enterprises had been relocated to Suzhou. Moreover, countylevel cities of Suzhou, namely, Kunshan, Changshu, Zhangjiagang, and Taicang, ranked high in the top 100 counties of China, injecting greaT vitality into Suzhou's economic development.

\section{CONCLUSIONS}

Regional city network has become an important tool to analyze the interaction and resource flow between cities. Many relevant studies have relied on social network analysis to identify features like node centrality, divide regional cities into different levels, or explore the relationship between a core city and the peripherical cities.

This paper constructs a regional city economic gravity network based on the gravity model of spatial economics. Then, the optimal tree theory of operations research was introduced to optimize the proposed network. Based on the 2018 indices (population, employment, and economy) of each city in the Yangtze River Delta, a regional city economic gravity network was established for the region, and optimized with the optimal tree approach. The optimal solution ensures the rational flow of resources at the regional level, and creates a mechanism for long-term circulation of resources.

The research findings challenge a widely accepted conclusion of the traditional research: the economic development of the Yangtze River Delta follows the pattern of 
one leader (Shanghai) and two wings (Nanjing and Hangzhou). The traditional conclusion overestimates the influence of administrative divisions, as Nanjing and Hangzhou are provincial capitals with greater political influence and population over prefecture-level cities in the region. Based on economic gravity connections, this paper identifies Suzhou as an economic subcenter that directly radiates nearby cities like Changzhou, Wuxi, and Huzhou. This finding reveals the current pattern of regional economic growth in the Yangtze River Delta. Apart from Shanghai, the role and potential of Suzhou in regional economic connectivity and regional integration cannot be underestimated.

Based on the evidence of this research, the location value of Suzhou in the economic pattern of the Yangtze River Delta will be presented in the future. Moreover, the future research will try to construct a mechanism to maximize long-term circulation of resources between cities in resource-deficient regions.

Drawing on the above conclusions, several policy suggestions were put forward:

(1) From the perspective of resource utilization, priority should be given to the key gravitational path on the optimal tree of the network. To formulate the overall circulation plan of a specific region, the efficient circulation path of limited resources must be addressed, in view of the scarcity of human, capital, and material resources. As in the case analysis, a circulation plan was formulated as per the optimal solution $T_{\max }$ of economic gravity network, which maximizes the total economic gravity of regional connectivity and the gravitational effect between cities. In this way, the resources in the region can flow rationally and be allocated efficiently, optimizing the resource utilization pattern.

(2) From the perspective of sustainable design, the collection and distribution effect of the optimal tree should be given full play. Low-carbon development and environmental protection have become the defining trends of our era. It is unwise for any regional city to pursue rapid growth at the cost of environment. Hence, eco-environmental sustainability must be considered in the circulation planning and utilization of regional resources. From the existing data on the cities in the Yangtze River Delta, the authors noted a largely positive correlation between economic scale and resource consumption. For instance, Shanghai, the central city with the best economic quality, faces huge pressures on public resources, including land scarcity, surging housing prices, and the lack of education and medical resources. When it comes to regional planning, the factors of resource consumption should be dispersed along the key gravitational path $T_{\max }$, making them more environmental friendly.

(3) From the perspective of industrial layout, the regional industrial structure on the optimal tree of the network should be optimized and upgraded. Owing to difference in location and history, the cities in the same region often vary in nature, type, grade, scale, and industrial structure. It is important to plan the industrial layout through overall consideration of the functions, division of labor, and positioning of each city in the network. For instance, cities at the core the Yangtze River Delta can extend their industrial chains along the key gravitational path $T_{\max }$ and make a long-term development plan for industrial optimization and transfer; the cities on the key gravitational path can attract industries transferred from the connected areas, according to the preferential policies and financial subsidies provided by the government. Such a plan will further rationalize the industrial layout of the cities in the region and enhance their industrial competitiveness, thereby improving the economic development of the entire region.

\section{REFERENCES}

[1] Peng, F.M. (2017). Economic spatial connection and spatial structure of Guangdong-Hong Kong-Macao greater bay and the surrounding area cities-an empirical analysis based on improved gravity model and social network analysis. Economic Geography, 37(12): 57-64.

[2] Lao, X., Shen, TY., Yang, Y., Zhang, Y. (2016). A study on the economic network of the urban agglomeration in the middle reaches of the Yangtze River: based on social network analysis method with gravity model. Urban Development Studies, 23(7): 91-98.

[3] Zhao, M.X. (2011). Evolution of network and spatial structure in Yangtze River Delta. Geographical Research, 30(2): 311-323. https://doi.org/10.11821/yj2011020011

[4] Wang, Q., Zhao, M. (2018). Research on the city network of Guangdong, Hongkong and Macao from the perspective of information flow: Analysis based on Baidu index. Journal of Regional and City Planning, 29(3): 281-293. http://dx.doi.org/10.5614\%2Fjrcp.2018.29.3.6

[5] Camagni, R.P. (1993). From city hierarchy to city network: reflections about an emerging paradigm. Structure and Change in the Space Economy, 66-87. https://doi.org/10.1007/978-3-642-78094-3_6

[6] Garcia, B.C., Chavez, D. (2014). Network-based innovation systems: A capital base for the Monterrey city-region, Mexico. Expert Systems with Applications, 41(12): $5636-5646$. https://doi.org/10.1016/j.eswa.2014.02.014

[7] Abelem, A.J.G., Stanton, M.A. (2005). Alternatives for community metropolitan networks for the major cities of the Amazon Region of Brazil: The case of Belem. 2nd International Conference on Broadband Networks, Boston, MA, pp. 1492-1498. https://doi.org/10.1109/ICBN.2005.1589781

[8] Derudder, B., Taylor, P.J., Hoyler, M., Ni, P.F., Liu, X.J., Zhao, M.X., Shen, W., Witlox, F. (2013). Measurement and interpretation of connectivity of Chinese cities in world city network, 2010. Chinese Geographical Science, 23(3): 261-273. https://doi.org/10.1007/s11769-0130604-y

[9] Derudder, B., Cao, Z., Liu, XJ., Shen, W., Dai, L., Zhang, W.Y., Caset, F., Witlox, F., Taylor, P.J. (2018). Changing connectivities of Chinese cities in the world city network, 2010-2016. Chinese Geographical Science, 28(2): 183-201. https://doi.org/10.1007/s11769-0180938-6

[10] Tinbergen, J. (1962). Shaping the World Economy: Suggestions for An International Economic Policy. New York: The Twentieth Century Fund.

[11] Pöyhönen, P. (1963). A tentative model for the volume of trade between countries. Weltwirtschaftliches Archiv, 90: 93-100. https://doi.org/10.2307/40436776

[12] Pitonak, M., Sprlak, M., Novak, P., Tenzer, R. (2017). Regional gravity field modelling from GOCE observables. Advances in Space Research, 59(1): 114127. https://doi.org/10.1016/j.asr.2016.09.024

[13] Xia, C., Zhang, A.Q., Wang, H.J., Zhang, B. (2019). Modeling urban growth in a metropolitan area based on 
bidirectional flows, an improved gravitational field model, and partitioned cellular automata. International Journal of Geographical Information Science, 33(5): 877-899. https://doi.org/10.1080/13658816.2018.1562067

[14] Alexander, N., Rhodes, M., Myers, H. (2011). A gravitational model of international retail market selection. International Marketing Review, 28(2): 183200. https://doi.org/10.1108/02651331111122669

[15] Fernandez, F.R., Hinojosa, M.A., Puerto, J. (2004). Multi-criteria minimum cost spanning tree games. European Journal of Operational Research, 158(2): 399408. https://doi.org/10.1016/j.ejor.2003.06.013

[16] Zhang, J.Y., Zhou, J., Zhong, S.Y. (2014). Models for inverse minimum spanning tree problem with fuzzy edge weights. Journal of Intelligent \& Fuzzy Systems, 27(5): 2691-2702. https://doi.org/10.3233/IFS-141384

[17] Fabijanska, A., Goclawski, J. (2014). New accelerated graph-based method of image segmentation applying minimum spanning tree. IET Image Processing, 8(4): 239-251. https://doi.org/10.1049/iet-ipr.2013.0104

[18] Hou, P., Hu, W.H., Chen, Z. (2016). Optimisation for offshore wind farm cable connection layout using adaptive particle swarm optimisation minimum spanning tree method. IET Renewable Power Generation, 10(5): 694-702. https://doi.org/10.1049/iet-rpg.2015.0340

[19] Tang, C., Hou, C.P., Wang, P.C., Song, Z.J. (2016).
Salient object detection using color spatial distribution and minimum spanning tree weight. Multimedia Tools and Applications, 75(12): 6963-6978. https://doi.org/10.1007/s11042-015-2622-5

[20] Li, H.W., Mao, W.J., Zhang, A.A., Li, C. (2016). An improved distribution network reconfiguration method based on minimum spanning tree algorithm and heuristic rules. International Journal of Electrical Power \& Energy Systems, 82(1): 466-473. https://doi.org/10.1016/j.ijepes.2016.04.017

[21] Han, X.Y., Meng, X.R., Kang, Q.Y., Su, Y.Z. (2019). Survivable virtual network link shared protection method based on maximum spanning tree. IEEE Access, 7: 92137-92150. https://doi.org/10.1109/ACCESS.2019.2927490

[22] Helmi, B.H., Rahmani, A.T. (2014). Maximum spanning tree based linkage learner. AI Communications, 27(3): 263-274. https://doi.org/10.3233/AIC-140594

[23] Lorenzo, L., Lorenzo-Freire, S. (2009). A chaFFcterization of Kruskal sharing rules for minimum cost spanning tree problems. International Journal of Game Theory, 38(1): 107-126. https://doi.org/10.1007/s00182-008-0147-0

[24] Kritikos, M., Ioannou, G. (2017). A greedy heuristic for the capacitated minimum spanning tree problem. Journal of the Operational Research Society, 68(10): 1223-1235. https://doi.org/10.1057/s41274-016-0146-7 